\title{
Delegierte Psychotherapie: Auflösung der SGDP
}

\section{Andreas Roose, \\ Christian Bernath}

Co-Präsidenten Schweizerische Ärztegesellschaft für Delegierte Psychotherapie (SGDP)
Korrespondenz:

Kommission Delegierte Psychotherapie

Kommissionssekretariat KDP Büro B

Hardturmstrasse 265

CH-8005 Zürich

Tel. 0432052955

kpd[at]psychiatrie.ch

www.delpsy.ch
Die Schweizerische Ärztegesellschaft für Delegierte Psychotherapie (SGDP) wurde 1999 vorerst unter dem Namen Interessengemeinschaft Delegierte Psychotherapie (IGDP) gegründet. Anlass dazu waren Versuche der Krankenkasse, eigene Kriterien für die Delegierte Psychotherapie aufzustellen und Positivlisten zu führen und andererseits war unklar, ob und wie die Delegierte Psychotherapie in den TARMED aufgenommen werden soll. Da die Delegierte Psychotherapie nicht die Domäne einer einzelnen Fachgesellschaft ist, sondern neben den Psychiatern auch von Allgemeinärzten, Internisten, Gynäkologen, Neurologen, Pädiatern, Rheumatologen usw. durchgeführt wird, war es sinnvoll, eine sowie Pychotherapeutinnen und Psychotherapeuten, die Etablierung eines Fähigkeitsausausweises für Nichtpsychiater und die tarifliche Einordnung sind abgeschlossen. Deshalb hat der Vorstand der SGDP den Antrag gestellt, die Gesellschaft aufzulösen und die Durchführung des Fähigkeitsausweises wieder an die FMPP zurückzugeben. Die heutigen Probleme im Zusammenhang mit der Delegierten Psychotherapie sind zum grossen Teil identisch mit denen der Psychiater. Die Generalversammlung der SGDP hat am 26. 3. 2012 den Antrag einstimmig angenommen. Das Vermögen der SGDP geht in einen speziellen Fonds bei der FMPP, der ausschliesslich für die Angelegenheit der Delegierten Psychotherapie zur

\section{Die Schweizerische Ärztegesellschaft für Delegierte Psychotherapie hat sich aufgelöst. Die Delegierte Psychotherapie wird in Zukunft durch eine entsprechende Kommission der Verbindung der psychiatrisch- psychotherapeutischen Ärztinnen (FMPP) vertreten.}

eigene Gesellschaft zu gründen. Sie war in der Folge auch Ansprechpartnerin für FMH, Kostenträger und Behörden. Inzwischen konnte ein Fähigkeitsauseis geschaffen werden und die Rahmenbedingungen für die Delegierte Psychiatrie wurden für die Spartenregelung des TARMEDs definiert.

Die FMH hat die Verbindung der psychiatrischpsychotherapeutisch tätigen ÄrztInnen (FMPP) mit der Verwaltung des Fähigkeitsausweises Delegierte Psychotherapie beauftragt. Sie hat auch festgelegt, dass dies durch eine Kommission zu geschehen habe, die paritätisch durch Psychiater und Somatiker besetzt ist und die ihre Entscheide einstimmig zu fassen habe. Die Kommission ihrerseits hat die konkrete Arbeit der Verleihung des Fähigkeitsausweises und der Rezertifizierung an die SGDP delegiert.

Die grosse Arbeit der Reglementierung der Delegierten Psychotherapie - die Festlegung der Anforderungen an die delegierenden Ärztinnen und Ärzte
Verfügung steht und durch die Kommission verwaltet wird.

Zurzeit besteht die Kommission Delegierte Psychotherapie aus folgenden Personen:

- Christian Bernath, Präsident, FMPP

- Lito Panayotopoulos, SGPP

- Hanspeter Bringolf, SGKJPP

- Andreas Minzer, SAPPM

- Jürg Sturzenegger, SGPRM

- Andreas Roose, KHM

\section{Kontakt}

Fragen im Zusammenhang mit der Delegierten Psychotherapie können entweder an das Sekretariat oder direkt an die Kommission gerichtet werden.

Die wichtigsten Unterlagen und Informationen zur Delegierten Psychotherapie werden weiterhin auf der Homepage www.delpsy.ch publiziert und laufend aktualisiert. 